\title{
Gradient effects in fracture mechanics for nano-structured materials
}

\author{
S. Lurie ${ }^{1, a}$, P. Belov ${ }^{2, b}$ \\ ${ }^{1}$ Institute of Applied Mechanics of RAS, Moscow, 119991, Leninskii 32a, Russia \\ ${ }^{2}$ GK “Rostechnology”, OAO “MMEZ-KT”, 123290, 1-th Magistralnyi pr.”, 9, Russia \\ asalurie@mail.ru, b p.belov@mepct.ru
}

Keywords: Gradient theory, adhesion model, cohesion field, equilibrium cracks, critical stresses, trajectories of stresses, scale parameter, J-integral.

\begin{abstract}
Gradient elastic effects associated with the existence of an cohesion type interphase layer, within a simple and robust gradient model are discussed. Classical and gradient solutions within a simple and robust fracture model whose properties are described by the harmonic and Helmholtz equations, are compared. It is shown that using the gradient solution can be explain the various mechanisms of fracture for the microcrystalline structures in dependence from scale parameter, which can be defined in terms of standard characteristics of materials. We established the equation for the tensor of energy, within a gradient model and adhesion model. Conservation law for the tensor of energy and J-integral equation were formulated for the continual gradient adhesion model.
\end{abstract}

\section{Introduction}

The development of continuum media models accounting for various micro/nanostructures beyond the theory of classical elasticity appears to be crucial for the description of not only short-range interactions and cohesion forces, but also for the modeling of other size-dependent effects in the framework of generalized elasticity and plasticity theories. Such robust gradient were developed initially by Aifantis and co-workers in the early eighties [1,2], for gradient plasticity and in the early ninties [3, 4] for gradient elasticity, respectively. Following the publication of Aifantis's initial models, various gradient theories appeared and applied to interface, shear banding dislocation and size effect and composite problems [5-7]. In the listed works the variants of gradient models for the description of cohesion scale-effect (without adhesive interactions) are developed.

It has been argued some time, that gradient theories may be quite effective to describe phenomenologically the influence of underlying microstructures, and have been used for capturing scale effects in miniaturized components and devices [7-9]]. In connections with the present works it is noted that Lurie and co-workes [10-14] have employed a first-order unified gradient model of the medium with conserved dislocations to describe a spectrum of various surface phenomena and scale effects, and an applied interphase layer model was also proposed [15-18]. These applied gradient models contain essentially only one additional physical parameter to account for straingradient effects; more so, in particular, since they also provide a sufficient description of the cohesion field with adhesion interactions in the contact zone between different components.

In this paper, using the gradient theory, we construct an analytical asymptotic solution for the model problem in the mechanics of fracture. We show that in contrast to the classical theory of gradient elasticity theory gives a non-singular solution for the crack. The novelty of the approach lies on the fact that the gradient theory allows us to construct asymptotic solutions of varying smoothness of the crack tip. In addition, we constructed an expression for the energy tensor and developed $\mathrm{J}$ integral to the case of the gradient theory, which taking into account cohesion and adhesion interactions. 
Preliminaries. The We examine a special case of Toupin's and Mindlin's theories of generalized elasticity [19,20,11,12,16,19], which was independently proposed and first elaborated upon by Aifantis [3]. The following variational model of the cohesion field is used:

$$
\delta L=\delta(A-E)=0, \quad E=\int_{G} E_{G} d V+\int_{\partial G} E_{\partial G} d V^{\prime}, A=\int_{G} A_{G} d V+\int_{\partial G} A_{\partial G} d V^{\prime}
$$

Here $A$ is the work of given forces in the volume $V$ and at the surface $V^{\prime}$; the total potential energy $(E)$ expressed as the sum of the integrals of the strain energy density in the volume $E_{G}$ and on the surface $\left(E_{\partial G}\right)$ of a gradient-dependent elastic material

$$
E_{G}=(1 / 2)\left[2 \mu \varepsilon_{i j} \varepsilon_{i j}+\lambda \theta^{2}+C u_{i} u_{i}\right], \quad E_{\partial G}=D_{i j} \dot{R}_{i} \dot{R}_{j} / 2
$$

Here $E$ is the total potential energy including the contribution from both the classical and gradient components of the strain energy, $R_{i}$ is the displacement field, $\varepsilon_{i j}=\left[R_{i},{ }_{j}+R_{j},{ }_{i}\right] / 2$ is the strain tensor, $\theta=R_{i, k} \delta_{i k}=\operatorname{div} \boldsymbol{R}$ is the spherical part of strain tensor, $\dot{R}_{i}=\left(\partial R_{i} / \partial x_{j}\right) n_{j}$ and $C$ is an additional physical constant of the model (the gradient coefficient) that determines cohesive interactions $[14,16,17]$ in the volume. The gradient term $u_{i}$ is defined as $u_{i}=-(1 / C) L_{i j}\left(R_{j}\right)$, with $L_{i j}(*)=\mu(*)_{, p p} \delta_{i j}+(\mu+\lambda)(*)_{, i j}$ being the classical Lamé

operator $\left(\mu, \lambda\right.$ are the Lamé coefficients and $\delta_{i j}$ is the Kronecker delta) [12,14-16], and $D_{i j}=A n_{i} n_{j}+B\left(\delta_{i j}-n_{i} n_{j}\right)$ determining the density of the strain energy on the surface; where the physical constants $A$ and $B$ are correspond to the normal and shear adhesion, $n_{i}$ is the outwards unit normal to the surface. Note, that in common case strain energy density on the surface $\left(E_{\partial G}\right)$ can define more common adhesion properties [11,12,16,19].

For the model of Eq. (1),(2) the system of the governing differential equations for the displacement vector $R_{k}$ can be written as:

$$
L_{i j} H_{j k}\left(R_{k}\right)+P_{i}^{G}=0 \quad L_{i j}(*)=\mu(*)_{, p p} \delta_{i j}+(\mu+\lambda)(*)_{, i j} \quad H_{j k}=(*) \delta_{j k}-L_{j k}(*) / C,
$$

The governing differential relations given by Eqs. (3) show that the displacement field can be written as a sum of two terms [16]: the classical component of the displacement field $U_{i}$ and the gradient part of the displacement field $u_{i}$ (cohesion-dependent component of the displacement field), i.e.

$R_{i}=U_{i}-u_{i}$ and $-C H_{i j}\left(u_{j}\right)+P_{i}^{G}=0$,

Here $u_{i}=-(1 / C) L_{i j}\left(R_{j}\right)$ and $U_{i}=H_{i j}\left(R_{j}\right)$. Note, that the classical Lamé operator can be defined as $L_{i j}\left(R_{j}\right)=E_{i j m k} R_{m, k j}, \quad C_{i j n m}=\lambda \delta_{i j} \delta_{n m}+\mu\left(\delta_{i n} \delta_{j m}+\delta_{i m} \delta_{j n}\right)$ and cohesion-dependent component of the displacement field takes the form $u_{i}=u_{i}=-(1 / C) L_{i j}\left(R_{j}\right)=-(1 / C) C_{i j m k} R_{m, k j}$. Then the strain energy density in the volume $E_{G}(2)$ can be written also in the following form 
$E_{G}=1 / 2\left[C_{i j n m} R_{i, j} R_{n, m}+\left(C_{i j r k} E_{n m r l} / C^{V}\right) R_{i, j k} R_{n, m l}\right]$

Full stresses $\sigma_{i j}=C_{i j n m} R_{n},{ }_{m}$ is the linear combination of classical stresses $s_{i j}$ and cohesion stresses $t_{i j}=C_{i j n m} u_{n},{ }_{m}$ Cohesion stresses are defined by the equilibrium equation $t_{i j},{ }_{j}-C u_{i}+P_{i}^{G}=0$ [14].

We will consider the fracture mechanical problem using the simplified formulation for the normal separation cracks. To receive more clear physical results we consider problem about opening crack in particular, simplified double plane formulation using the following propositions: $R_{i} Y_{i}=R(x, y)$, a $R_{i} X_{i} \equiv 0,\left\{X_{i}\right\},\left\{Y_{i}\right\}$ are the vectors of coordinate axis. Assume also that density of the forces in the volume is equal to zero $\boldsymbol{P}^{G}=0$. To receive clear physical results we consider problem about opening crack in simplified double plane formulation using the following propositions respect to the displacement field: $R_{i} Y_{i}=R(x, y)$, and $R_{i} X_{i} \equiv 0$. Here $\left\{X_{i}\right\},\left\{Y_{i}\right\}$ are the vectors of coordinate axis. In this case we have the following equation for the density of strain energy

$$
E_{G}=(1 / 2)\left[\mu R,_{x} R,_{x}+(2 \mu+\lambda) R,_{y} R,_{y}+C u u\right]
$$

Can be shown, that for this scalar problem classical part of solution $U(x, y)$ satisfies the harmonic equation $\nabla^{2} U=0, \quad \nabla^{2}(\ldots)=\partial^{2}(\ldots) / \partial \bar{x}^{2}+\partial^{2}(\ldots) / \partial y^{2}, \quad \bar{x}=x \sqrt{(2 \mu+\lambda) / \mu} \quad$ and cohesion part $u=u(\bar{x}, y)$ satisfies the Helmholtz equation $l_{E}^{2} \nabla^{2} u-u=0, l_{E}=\sqrt{(2 \mu+\lambda) / C}$. Scalar field of defects is satisfies to the governing equation of the four order, i.e. $\nabla^{2}\left[l_{E}^{2} \nabla^{2} R-R\right]=0$.

For the model under consideration the component stresses in the X-direction is zero. Normal stresses $\sigma$ along axis $\mathrm{OY}$ and shear stresses $\tau$ can be written in the following form $\sigma=(2 \mu+\lambda) R_{, y}=(2 \mu+\lambda)\left[U_{, y}-u_{, y}\right], \quad \tau=\mu R_{, x}=\mu\left[U_{, x}-u_{, x}\right]$

Classical solution. We consider problem about opening crack in particular, simplified plane formulation. The homogeneous boundary conditions have place on the crack faces $(x \geq 0, y=0)$. The following asymptotic solution for displacement and stresses near top of crack can be written:

$$
U(r, \varphi)=2 K_{I} r^{1 / 2} \operatorname{Cos}(\varphi / 2) /(2 \mu+\lambda), \quad \sigma=K_{I} r^{-1 / 2} \operatorname{Sin}(\varphi / 2)
$$

Suppose that at some point of the body the stresses becomes equal to the ultimate strength $\sigma=\sigma_{c}$. Then the stress intensity factor $K_{I}$ must be equal to the coefficient of fracture toughness $K_{I}=K_{I c}$. Using equation (7) we can find the trajectory of the critical stresses $\sigma_{c}$

$$
r_{c}(\varphi)=\left(K_{I c} / \sigma_{c}\right)^{2} \operatorname{Sin}^{2}(\varphi / 2)
$$

Averaging the function $r_{c}=r_{c}(\varphi),(8)$ of the angle, we obtain the equation for the critical grain size $d$; i.e.

$$
d / 2=(2 \pi)^{-1} \int_{0}^{2 \pi} r_{c}(\varphi) d \varphi=(1 / 2)\left(K_{I c} / \sigma_{c}\right)^{2}
$$


Allowing the equation (9) with respect to stresses, we can establish the Hall-Petch law in fracture

$$
\sigma_{c}=K_{I c} d^{-1 / 2}
$$

We can offer the following interpretation of the obtained results: in the case of brittle material the fracture mechanism is such that at the front of the crack the grains are crumbled from material with a typical diameter $d,(9)$, and with shapes that define by equation (8). Let's consider the trajectories (8), having a common point at the crack tip. We assume that the trajectory is critical when $\mathrm{r} r=d$. The solutions based on the classical theory of elasticity can be used if $r \geq d$. The critical grain size $d$ determines the area of applicability of the classical theory of elasticity. On the other hand, non classical effects can occur for regions whose diameter is less then critical $r<d$. The Hall-Petch low is one of the examples of the non-classical effects which depend from the characteristic scale parameter of structure [14]. Systematic deviations from the Hall-Petch law can take place for the small grain size.

Gradient solution in the fracture. For the gradient theory the stress state problem for opening crack is constructed as linear combination of classical $(s)$ and cohesion $(t)$ stresses[14]: $s=K_{I}\left(l_{E}\right)^{-1 / 2} z^{-1 / 2} \operatorname{Sin}(\varphi / 2), \quad t=K_{I}\left(l_{E}\right)^{-1 / 2}(2 / \pi)^{1 / 2} K_{1 / 2}(z) \operatorname{Sin}(\varphi / 2)$, where $K_{I}$ is the stress intensity factor, angle $\varphi$ counts out from positive axis $x, K_{1 / 2}(z)$ is the cylindrical function of Macdonald with indexes equal 1/2. This solutions are satisfied the homogeneous boundary conditions on the crack faces $(x \geq 0, y=0)$. Taking into account known asymptotical properties of the Macdonald function $K_{1 / 2}(z)$ with $|z| \rightarrow 0$, we can conclude that function $t=K_{I}\left(l_{E}\right)^{-1 / 2}[\exp (-z) / z] \operatorname{Sin}(\varphi / 2)$ describes the cohesion type stress field near top of crack. Then, using mention above solutions $s(r, \varphi), t(r, \varphi)$, it is easy to find the nonsingular solution with the classical asymptotical behavior on the infinity $(r \rightarrow \infty)$; i.e.

$$
\sigma=K_{I}\left(l_{E}\right)^{-1 / 2}\left(1-e^{-z}\right)(z)^{-1 / 2} \operatorname{Sin}(\varphi / 2), z=r / l_{E}, l_{E}^{-2}=C /(2 \mu+\lambda)
$$

Note, that an upper and lower face of crack are correspond to the angles $\varphi=0, \varphi=2 \pi$. It is easy to show that asymptotic solution (12) gives nonsingular stresses field near top of crack and nonclassical dependences for the displacement of crack faces similar to the dependences for equilibrium cracks introduced by Barenblatt. The displacement of crack faces can be found by integrating of equations for stresses $\sigma$ (12); i.e. $R(r, \varphi)=2 K_{I}\left(l_{E}\right)^{1 / 2}(2 \mu+\lambda)\left(1-e^{-z}\right)(z)^{1 / 2} \operatorname{Cos}(\varphi / 2)$. It is easy to see, that the angle between bounds at the crack tip is equal to zero as it takes place and for equilibrium cracks. Let us consider the trajectories of critical stresses for gradient solution (12);i.e.

$$
z^{1 / 2} /\left(1-e^{-z}\right)=q \operatorname{Sin}(\varphi / 2), \quad q=\left[K_{I c} /\left(l_{E}^{1 / 2} \sigma_{c}\right)\right]
$$

It can be shown that it exists such small parameter $q$, when the bundle of trajectories (13) near tip of crack has place. In this case there are not roots of the equation (13) for $\varphi=\pi$. Thus, the closed subarea with the critical size of a grain is allocated. This trajectory of critical stresses gives the forecast of area of destruction. 
Gradient solution of high smoothness. We consider the simplified model (1),(2),(6) with governing equation $\nabla^{2}\left[l_{E}^{2} \nabla^{2} R-R\right]=0$. Let us show that it is possible to construct the variant gradient nonsingular solution with high smoothness near the top of the crack. For the opening crack with the homogeneous boundary conditions on the crack faces $(x \geq 0, y=0)$ the following solution for the displacement can be written:

$$
R(z, \varphi)=l_{E}\left\{A_{1} z^{-1 / 2}-C_{1} K_{1 / 2}(z)+2 K_{I} l_{E}^{-1 / 2} z^{1 / 2} /(2 \mu+\lambda)\right\} \operatorname{Cos}(\varphi / 2)
$$

Then we can find equation for the stresses:

$$
\begin{aligned}
& \sigma(z, \varphi)=\left\{\left[-A_{1} z^{-3 / 2}+C_{1} K_{3 / 2}(z)\right] \operatorname{Sin}(3 \varphi / 2)+\right. \\
& \left.+\left[2 K_{I} l_{E}^{-1 / 2} z^{-1 / 2} /(2 \mu+\lambda)+C_{1} K_{1 / 2}(z)\right] \operatorname{Sin}(\varphi / 2)\right\}(2 \mu+\lambda) / 2
\end{aligned}
$$

Here $K_{1 / 2}(z)$ и $K_{3 / 2}(z)$ are the cylindrical Macdonald functions of half-integer order. To satisfy the regularity conditions of the displacement at the crack tip we can put in (15): $A_{1}=C_{1} \sqrt{\pi / 2}$. Then, using properties of the Macdonald functions we can receive:

$$
R(z, \varphi)=\left[2 K_{I} z^{-1 / 2} l_{E}^{1 / 2} /(2 \mu+\lambda)\right]\left(e^{-z}-1+z\right) \operatorname{Cos}(\varphi / 2)
$$

In this case it is easy to verify that the stresses will also be regular at the crack tip:

$$
\left.\sigma(z, \varphi)=K_{I} l_{E}^{-1 / 2} z^{-3 / 2}\left(1-e^{-z}-z e^{-z}\right) \operatorname{Sin}(3 \varphi / 2)+\left(1-e^{-z}\right) z^{-1} \operatorname{Sin}(\varphi / 2)\right\}
$$

Note, that in contrast to classical fracture mechanics where angle at the crack tip is equal 180 degrees, for the nonclassical gradient theory (16) this angle equal to zero. We can see also that dependence $R(z, \varphi=0)$ has a inflection point in a vicinity of top of a crack. It is noteworthy that the distance from the inflection point to the crack tip is almost identical to the non-classical parameter of the model $l_{E}$. So, we can treat this parameter in fracture mechanics as a characteristic parameter of the materials.

Let us consider the trajectories of the critical stresses for the gradient solution equal stresses. Introducing the critical parameter $q=K_{I c} /\left(\sigma_{c} l_{E}^{1 / 2}\right)$ with the aid of equation (17) we can write the following implicit equation for the critical stresses trajectory:

$$
f(z, \varphi, q)=0
$$

Where $f(z, \varphi, q)=q z^{-1 / 2}\left\{\left[\left(1-e^{-z}\right)-z e^{-z}\right] \operatorname{Sin}(3 \varphi / 2)+\left(1-e^{-z}\right) \operatorname{Sin}(\varphi / 2)\right\}-1$

Fig. 1 shows trajectories of stresses for the gradient solution (18), which are depended from parameter $q=K_{I c} /\left(\sigma_{c} l_{E}^{1 / 2}\right)$.

The trajectories of the critical stress as well as in the classical case, identify the area in which the stresses exceeds a critical value. If the parameter $z^{-1}<<1$, then the equation of the trajectory of the critical stress (18) gives the trajectory of the classical solution, $z=\left(K_{I c} / \sigma_{c} l_{E}^{1 / 2}\right)^{2} \operatorname{Sin}^{2}(\varphi / 2)$ (see also Eq. (14)). Thus, for sufficiently large values of parameter $z$ the classical and gradient solutions are coincided up to $O\left(z^{-1}\right)$. As noted earlier the characteristic size of this area is $d=\left(K_{I c} / \sigma_{c}\right)^{2}$. 


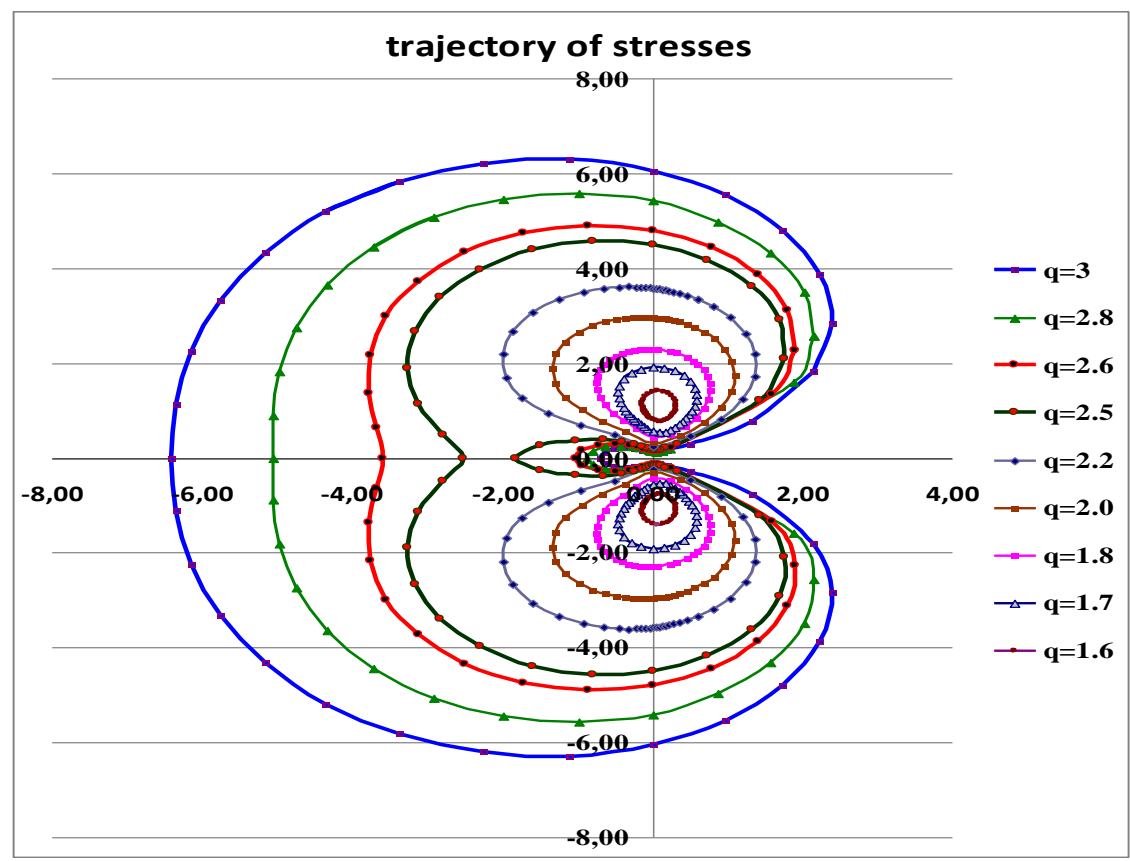

Fig.1 Trajectories of stresses for gradient solution

Let's note, that for $\varphi=\pi$, there is such value of parameter $q=q_{c}$, when the equation (18) has only one root in contrast of the classical case when equation (18) has two roots. In this case two zones are allocated instead of one critical subarea. Physically it means, that the critical border of failure lies on border of two neighbor grains. It is possible to show, that for a considered problem parameter $q_{c}$ is equal $q_{c}=2.489$. This parameter determines the changing of the fracture mechanism. To this value of characteristic parameter $q_{c}$ there corresponds a unique root $z_{c}=2.149$. Hence, the trajectory of critical stresses is closed in a case if $q>q_{c}$. At $q=q_{c}$ the trajectory has one root and became self crossed. At $q<q_{c}$ two, top and bottom subareas take place, symmetric concerning axis OX. In this case there are not roots of the equation (18) at $\varphi=\pi$. Recall that the similar result we received for the gradient model (12),(13) where only one critical subarea was allocated.

So, we are shown, that specified gradient solutions in the mechanics of fracture to the full corresponds to classification of nanoctructured materials [21]. This classification divides a dimensional set of nanocrystals on three groups: "big", "average" and "small" in which the fracture processes are defined by various mechanisms: a) fracture areas are defined by a characteristic sizes of grains; б) the fracture is determined by borders of grains; the area of fracture is supervised by joints of grains.

Conservation law of the tensor of energy for the gradient adhesion model. So, we shown, that the gradient model specifies our representation about mechanisms of fracture. Further we shall prove the theorem about the law of conservation of the tensor of energy and we shall establish the relations, allowing to formulate $\mathrm{G}$ integral for the gradient models which are taking into account and adhesive properties of the solids.

Let's receive equations for the tensor of energy and vector of a stream of the energy, taking into account cohesion interactions (gradient effects) in volume and adhesive interactions on the surface of the body. Using equation (1) (2), we can write Euler's equations in the form: 
$\partial A_{G} / \partial R_{i}+\left\{\partial E_{G} / \partial R_{i, j}-\left[\partial E_{G} / \partial R_{i, j k}\right]_{, k}\right\}_{, j}=0$

Assume that volume density of forces $P_{i}^{G}$ are not depended from the coordinates;i.e. $A_{G}\left(R_{i}\left(x_{j}\right), P_{i}^{G}\right)$. Multiplying last equation on $R_{i, p}$ and, using integration in parts we can find:

$$
\left[\left(A_{G}-E_{G}\right) \delta_{p q}+\left(\partial E_{G} / \partial R_{i, q}\right) R_{i, p}+\left(\partial E_{G} / \partial R_{i, j q}\right) R_{i, j p}-R_{i, p}\left(\partial E_{G} / \partial R_{i, j q}\right)_{, j}\right]_{, q}=0
$$

Expression in square brackets has physical sense of the tensor of energy.

$$
T_{p q}=\left(A_{G}-E_{G}\right) \delta_{p q}+\left[\partial E_{G} / \partial R_{i, q}-\left(\partial E_{G} / \partial R_{i, j q}\right)\right]_{, j} R_{i, p}+\left(\partial E_{G} / \partial R_{i, j q}\right) R_{i, j p}
$$

If the density of the potential energy in the volume $E_{G}=E_{G}\left(R_{i, j} ; R_{i, j k}\right)$ is independent of the curvature of the deformations $\partial E_{G} / \partial R_{i, j q}=0$, the tensor of energy $T_{p q}$ takes the classical form[8]:

As result, we can establish the differential conservation law of the energy tensor for the gradient theory; i.e. $T_{p q, q}=0$. It is easy to write the integral conservation law of the energy tensor for the gradient theory:

$$
\iiint T_{p q, q} d V=\iint T_{p q} n_{q} d F=\iiint T_{p} d F=0
$$

where $T_{p}=T_{p q} n_{q}=\left(A_{G}-E_{G}\right) n_{p}+\left\{\left[\left(\partial E_{G} / \partial R_{i, q}-\left(\partial E_{G} / \partial R_{i, j q}\right)_{j}\right] R_{i, p}+R_{i, j p} \partial E_{G} / \partial R_{i, j q}\right\} n_{q}\right.$

In the equation () the integration is over for any surface bounding the volume of the body. If the components of the stress or displacement are given on the body surface, the integral conservation law has a specific form, which is determined by the own surface potential energy $E_{\partial G}$ and the energy flux vector $T_{p}$ can be written as follows

$$
\left.\iint T_{p} d F=\iint A_{G}-E_{G}+\left(\partial A_{\partial G} / \partial R_{i}\right) \dot{R}_{i}-\left(\partial E_{\partial G} / \partial R_{i, j}\right) \dot{R}_{i, j}\right) n_{p} d F+\sum f\left(A_{F}-E_{\partial G}\right) v_{p} d s=0
$$

where $\delta_{k p}^{*}=\left(\delta_{k p}-n_{k} n_{p}\right), \dot{R}_{i}=\left(\partial R_{i} / \partial x_{j}\right) n_{j}, n_{j}$ is a normal vector to the surface.

As result we received equation which is the generalization of the G-integral for the gradient model which taking into account the adhesion interactions.

\section{Summary}

We have shown that the greater smoothness of the solution makes it possible to specify the configuration of regions of limit stress, depending on the characteristic size of the grain microstructure and its correlation with the scale parameter of gauge theories. As a result, proposed a classification of micro-and nanocrystalline structures, depending on the dominant type of fracture. In addition, the energy tensor was constructed and equation for the $\mathrm{J}$ integral was established for the gradient adhesion model

Acknowledgement. This work is supported by the Russian Foundation for Basic Research (N 12-0100273, 10-07-00040) and Program of the RAS PR21 


\section{References}

[1] E.C. Aifantis,Trans ASME. J. Engng. Mat. Tech. Vol.106; (1984), p. 326-330.

[2] E.C. Aifantis 1987. The physics of plastic deformation. Int. J. Plastisity, 3, 211-247

[3] E.C. Aifantis, Int. J. Engng. Sci. Vol. 30, (1992.), p.1279-1299.

[4] E.C. Aifantis, J. Mech. Behav. Mater. Vol. 5, (1994), p. 335-353.

[5] E.C. Aifantis, Int. J. Fract. Vol. 95, (1999), p. 299-314.

[6] N.A. Fleck, J.W. Hutchinson, J. Mech. Phys. Vol. 9, (2001), p. 2245-2271.

[7] K.E. Aifantis, J.R. Willis, J. Mech. Phys. Solids. Vol. 53, (2005), 1047-1070.

[8] R.D. Mindlin, J. Elasticity. Vol. 2(4), (1972), p.217-280.

[9] G.A. Maugin,.Appl. Mech. Rev. Vol. 48,( 1995), p. 213-24.

[10] S.Lurie, A. Kalamkarov, Int. J. Solids and Structures, Vol. 44, (2007), p. 7468-7485.

[11] P.A. Belov, S.A. Lurie, Appl. Math. and Mech. Vol. 73 (5), (2009), p. 833-848.

[12] S.A. Lurie, P.A.Belov, N.P. Tuchkova, In: One hundred years after the Cosserats. Series: Advances in Mechanics and Mathematics, Vol. 21. Maugin, G.A., Metrikine, A.V. (Eds.).1st Ed., XIX, Springer. 223-232, (2010).

[13] S.A. Lurie, P.A.Belov, N.P. Tuchkova, D.B. Volkov-Bogorodsky, Comp. Mater. Sci. Vol 28(3-4),( 2003), p. 529-539

[14] S.A. Lurie, P.A. Belov, Int. J. Fract., Vol. 50(1-2), (2008), p.181-194

[15] S.A. Lurie, P.A.Belov, N.P. Tuchkova, J. Composites. Part A: Vol. 36, (2005), p. 145-152.

[16] S.A. Lurie, D.B. Volkov-Bogorodsky, V.I. Zubov, N.P. Tuchkova, Int. J. Comp. Mater. Sci. Vol. 45(3),( 2009), p. 709-714.

[17] D. Volkov-Bogorodsky, Y. Evtushenko, I. Zubov, S. Lurie, J. Comput. Math. Math. Phys., Vol. 46 (7), (2006), p.1318-1337.

[18] S.A. Lurie, N.P. Tuchkova, Composites and nanostructures Vol. 2, (2009), p. 25-43.

[19] R.D. Mindlin, Arch. Rat. Mech. Anal. Vol.16, (1964), p. 51-58.

[20] R.A. Toupin, Arch. Ration. Mech. Analysis. Vol.17(2), (1964), p. 85-112.

[21] A.Gleser, Deformation and Fracture of Materials Vol.2 (2010), p.7-19 